\title{
INFLUÊNCIA DAS ESPÉCIES IÔNICAS DISSOLVIDAS NA FLOTAÇÃO DE CROMITA COM OLEATO
}

\author{
C. A. M Baltar ${ }^{1}$; T. A. Magalhães Filho ${ }^{2}$ e A. R. S. Medeiros ${ }^{2}$
}

${ }^{1}$ Grupo de Tecnologia Mineral - Universidade Federal de Pernambuco e ${ }^{2}$ PPG Eng. Mineral, UFPE camb@ufpe.br - tiagoamf@yahoo.com.br - ailma.engminas@gmail.com

Artigo submetido em outubro/2012 e aceito em novembro/2012

\section{RESUMO}

O minério de cromo da Bahia é constituído por cromita $\left(\mathrm{FeO} . \mathrm{Cr}_{2} \mathrm{O}_{3}\right)$ e minerais de ganga como: magnetita, serpentinitos e piroxênios. A flotação seletiva é bastante complicada devido às características superficiais similares dos componentes mineralógicos. Além disso, essas espécies lançam, na polpa, uma considerável quantidade de íons que interferem fortemente no resultado da flotação.

Nesse trabalho verificou-se a influência das espécies iônicas dissolvidas, na ação do coletor, e a possibilidade de se conseguir seletividade em flotação com oleato de sódio. Os resultados sugerem a formação de compostos/precipitados, na forma de carboxilatos que apresentam ação fortemente depressora para concentrações de coletor de até $10^{-4} \mathrm{M}$. A recuperação da cromita pode diminuir até $42 \%$ em relação à obtida em água destilada.

PALAVRAS-CHAVE: flotação de cromita, qualidade da água, íons dissolvidos, oleato de sódio, formação de sais carboxílicos.

\section{INFLUENCE OF DISSOLVED IONIC SPECIES ON CROMITE FLOTATION WIT OLEATE}

\section{ABSTRACT}

The chrome ore from Bahia consists of chromite $\left(\mathrm{FeO} . \mathrm{Cr}_{2} \mathrm{O}_{3}\right)$ and gangue minerals such as magnetite, serpentine and pyroxene. The selective flotation is quite complicated due to similar mineralogical components' surface characteristics. Furthermore, these species in the pulp release a considerable amount of ions that strongly influence the flotation results.

In this work, it was used techniques such as conductivity determination to monitor the presence of dissolved species in the water and their influence with sodium oleate on the system.
It was observed that dissolved species in the pulp such as $\mathrm{Ca}++, \mathrm{Mg}++$ and $\mathrm{Fe}+++$, consume part of the collector hindering flotation of chromite.

Tests were conducted on a Hallimond tube modified to demonstrate the detrimental effect of these dissolved species in the pulp on the flotation results.

KEY-WORDS: chromite flotation, chemical environment, dissolved ions, sodium oleate, carboxylic salts. 


\section{INFLUÊNCIA DAS ESPÉCIES IÔNICAS DISSOLVIDAS NA FLOTAÇÃO DE CROMITA COM OLEATO}

\section{INTRODUÇÃO}

A quebra da partícula no moinho proporciona a formação de novas superfícies com energia livre resultante da ruptura de ligações entre átomos. Devido à energia livre superficial, a superfície interage com moléculas de água e outras espécies em solução provocando a dissolução parcial do mineral, com liberação de espécies iônicas para a polpa (BALTAR, 2010).

Esses íons em solução, em geral, participam ativamente do processo de flotação atuando em diferentes frentes: alteração da carga elétrica superficial (RAO et al., 1988), precipitação do coletor, competição com o íon coletor pelos sítios disponíveis na superfície (BALTAR e CUNHA, 2002), estabilização da espuma (QUINN et al., 2007), etc.

Por sua vez, os coletores carboxílicos apresentam grande afinidade pelos alcalinos terrosos com os quais formam sais praticamente insolúveis. Dessa forma, a presença de espécies iônicas dissolvidas na polpa pode consumir parte das moléculas do coletor diminuindo a densidade de adsorção na superfície e, consequentemente, tornando a flotação mais lenta com reflexo na recuperação.

A influência de espécies catiônicas na flotação de diferentes minerais com amina foi estudada por RAO et al. (1988), BALTAR e CUNHA (2002), e PINHEIRO et al. (2010). RAO et al. (1988) conseguiram melhorar os resultados de uma flotação de pirocloro após a remoção de íons $\mathrm{Ca}^{2+}$ da água de reciclagem. BALTAR e CUNHA (2002) observaram que a presença de apenas 5 ppm de $\mathrm{Al}^{3+}$ reduziu a recuperação de feldspato de $95 \%$ para $50 \%$, enquanto o efeito depressor do $\mathrm{Ca}^{2+}$ e do $\mathrm{K}^{+}$só foi percebido para concentrações maiores do que $2000 \mathrm{ppm}$. A recuperação do quartzo, em flotação com dodecilamina, também depende fortemente da concentração das espécies catiônicas em solução (PINHEIRO et al., 2010). Os autores usaram uma água com elevado teor de cálcio e magnésio, e compararam os resultados antes e depois do tratamento da água para a remoção desses cátions, obtendo recuperações de $14 \%$ e $84 \%$, respectivamente, para a água dura e a água tratada.

A influência de espécies iônicas dissolvidas na polpa também tem sido estudada na flotação aniônica com coletores carboxílicos. NANTHAKUMAR et al. (2009) conseguiram melhorar a flotação de um minério de fosfato com "tall oil" usando carbonato de sódio para precipitar os íons $\mathrm{Ca}^{2+}$.

O estudo teve por objetivo verificar a influência de espécies catiônicas $\left(\mathrm{Ca}^{2+}, \mathrm{Mg}^{2+}, \mathrm{Fe}^{2+}, \mathrm{Al}^{3+} \mathrm{e}\right.$ $\mathrm{Cr}^{3+}$ ) provenientes da dissolução de superfícies minerais presentes na polpa, na ação do oleato de sódio na flotação da cromita. A velocidade de liberação das espécies iônicas foi monitorada através da determinação da condutividade da polpa. Os resultados mostraram que para concentrações de oleato de até $10^{-4} \mathrm{M}$, a presença dos íons em solução tem efeito bastante prejudicial na flotação da cromita, diminuindo a recuperação da cromita em até $42 \%$. 


\section{EXPERIMENTAL}

\section{$\underline{\text { Material }}$}

a) Amostra - As amostras de cromita e dos minerais de ganga foram fornecidas pela FERBASA.

b) Principais Equipamentos - Os testes de flotação foram realizados em Tubo de Hallimond modificado com diâmetro de $3,5 \mathrm{~cm}$ e constituído de três partes (Figura 1); agitador magnético QUIMIS modelo 241-2; condutivímetro INOLAB; pHmetro digital da DIGIMED, modelo DM-22.

c) Reagentes - Usou-se um oleato de sódio produzido a partir do ácido oleico e hidróxido de sódio, ambos os reagentes foram fabricado pela QUIMIBRAS.

\section{Metodologia}

\section{a) Testes de Flotação}

Os ensaios de microflotação foram realizados em tubo de Hallimond modificado (Figura 1) alimentado com uma vazão de nitrogênio de $80 \mathrm{~mL} / \mathrm{min}$. Usou-se 1 grama de cromita pura (faixa granulométrica $100 \%$ menor que $74 \mu \mathrm{m}$ ). $\mathrm{O} \mathrm{pH}$ foi fixado em 9,5 usando-se o hidróxido de sódio ( $\mathrm{NaOH}$ ) como regulador. Como coletor usou-se o oleato de sódio. A solução de oelato de sódio foi preparada a partir do acido oléico (fabricado pela QUIMIBRAS). O tempo de condicionamento foi de 1 minuto, e o tempo de flotação foi de 2 minutos. Em seguida o material foi filtrado, seco e pesado.

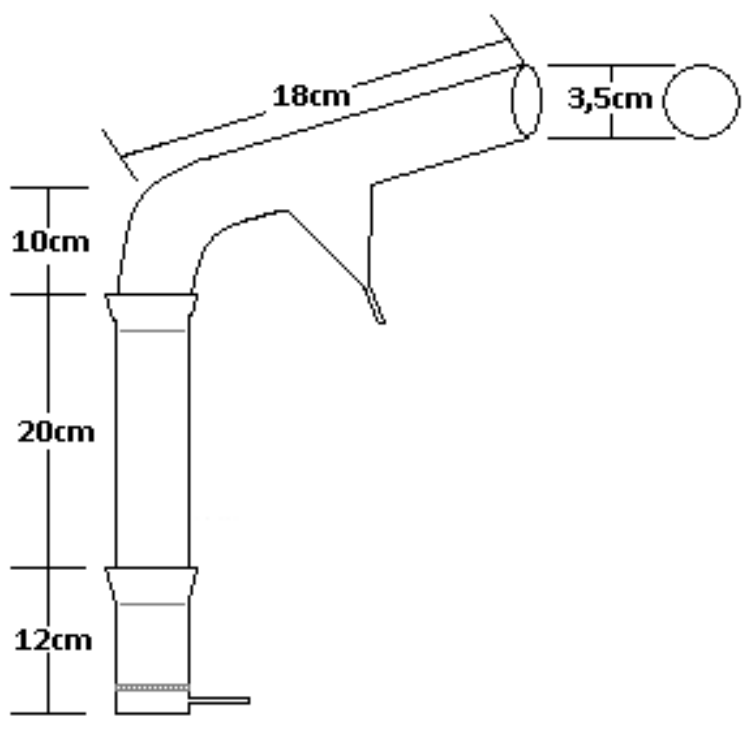

Figura 1 - Ilustração com as dimensões do Tubo de Hallimond modificado usado nos testes de flotação.

b) Condutividade

A polpa foi agitada por 1 minuto e, em seguida, deixada em repouso por mais 1 minuto. Uma amostra do sobrenadante foi retirada para a determinação da condutividade. 0 procedimento repetiu-se para cada teste. 
A água bidestilada usada nos testes teve condutividade igual a $2 \mu \mathrm{S} \cdot \mathrm{cm}^{-1}$, para efeito comparativo, a condutividade de uma amostra de água de torneira foi medida, sendo igual a, $467 \mu \mathrm{S} \cdot \mathrm{cm}^{-1}$.

Nos testes com oleato de sódio, a polpa foi agitada em água bidestilada por 6 minutos. Após decantar por 2 minutos, o sobrenadante foi misturado com oleato de sódio por mais 6 minutos, antes da determinação da condutividade.

c) Remoção dos Eletrólitos ("lavagem”)

Inicialmente, a polpa (com $20 \%$ de sólidos) foi agitada a $450 \mathrm{rpm}$, por 10 minutos. Em seguida, após dois minutos de decantação, retirou-se uma alíquota do sobrenadante para determinação da condutividade. O material restante foi filtrado e a torta resultante foi usada para a formação de nova polpa com água bidestilada, repetindo-se o ciclo de agitação/filtragem e adição de água nova, até a quinta "lavagem".

\section{RESULTADOS E DISCUSSÃO}

A concentração de eletrólitos na polpa pode ser monitorada através da determinação da condutividade. A Figura 2 mostra a variação da condutividade, com o tempo, para a água da torneira e para a água destilada antes e depois do contato com a cromita e com os minerais de ganga.

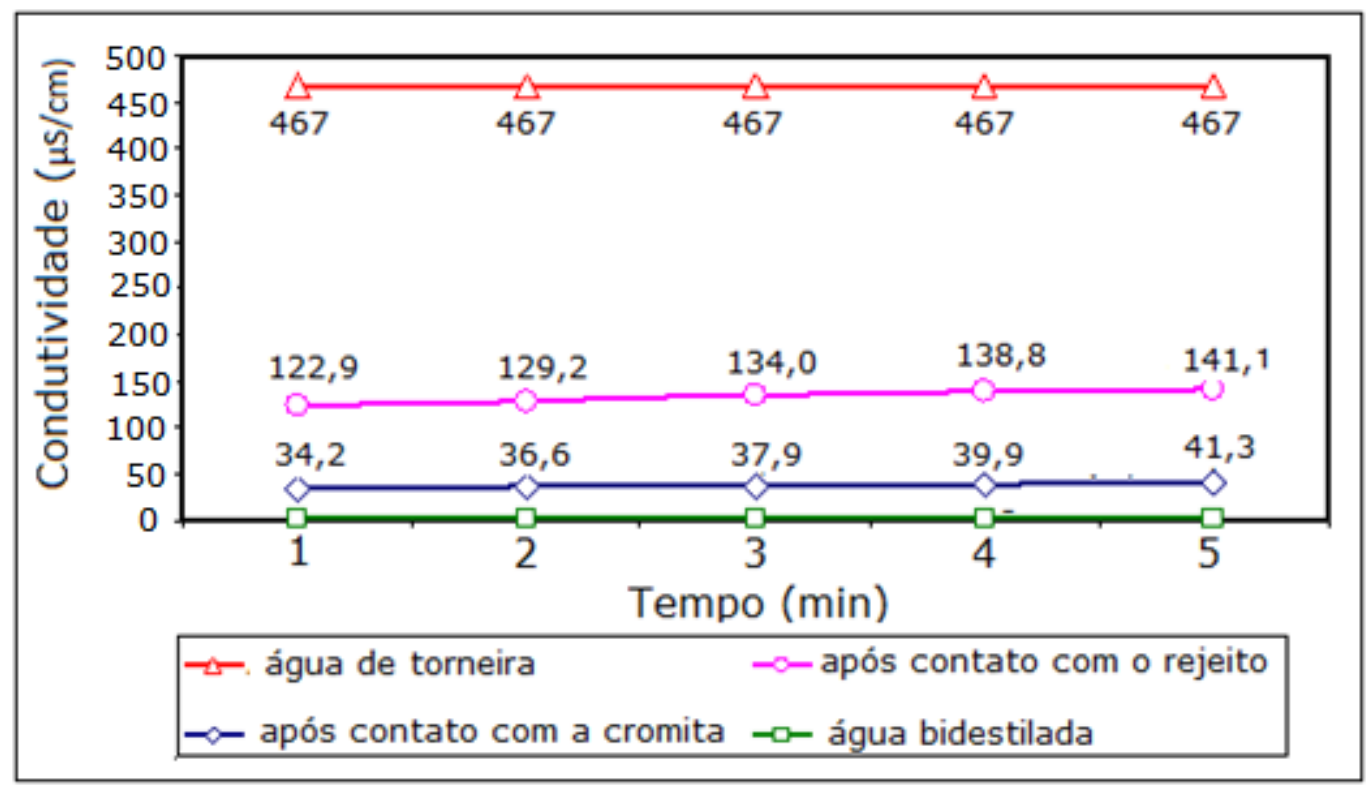

Figura 2 - Variação da condutividade com o tempo para águas com diferentes conteúdos de sais.

O aumento da condutividade verificado após o contato com o minério (Figura 2) confirma a liberação de espécies iônicas da superfície em contato com a água. Como era de se esperar, a condutividade da água destilada e da água de torneira manteve-se constante durante o período da agitação. Ao contrário, em contato com o minério, a condutividade aumentou à medida que prosseguiu o contato água-sólido, sugerindo a continuidade do processo de dissolução superficial. A velocidade de liberação dos íons na polpa, no entanto, 
diminui com o tempo. A dissolução é mais intensa nos minerais de ganga, a condutividade da polpa chegou a $141 \mu \mathrm{S} / \mathrm{cm}$ após 5 minutos de contato. A dissolução da cromita é mais lenta atingindo o máximo de $41,3 \mu \mathrm{S} / \mathrm{cm}$ no mesmo período de contato. Considerando-se que a condutividade original era de apenas $2 \mu \mathrm{S} / \mathrm{cm}$, houve um aumento considerável da concentração de eletrólitos na polpa que, no entanto, não atingiu o patamar da água de torneira $(467 \mu \mathrm{S} / \mathrm{cm})$.

A influência das espécies iônicas dissolvidas na flotação da cromita foi observada nos testes de flotação realizados em Tubo de Hallimond. A Figura 3 mostra a recuperação de cromita em função da concentração do coletor. Observa-se que para concentrações de oleato de até $2,5 \times 10^{-4} \mathrm{M}$, a recuperação da cromita diminui, significativamente, com o uso da água contaminada, ou seja, na presença das espécies dissolvidas. A queda na recuperação chegou a $42 \%$ no teste realizado com $5 \times 10^{-5} \mathrm{M}$ de oleato.

Esse fato pode ser explicado pela formação de sais carboxílicos, provavelmente, de $\mathrm{Ca}^{2+}, \mathrm{Mg}^{2+}$ e $\mathrm{Fe}^{2+}$ que consomem parte das moléculas do coletor, resultando em menor densidade de adsorção na superfície e, consequentemente, em menor recuperação. Para maiores concentrações de oleato, no entanto, a presença das espécies dissolvidas não interfere no resultado da flotação devido à maior disponibilidade de moléculas do coletor para o recobrimento superficial.

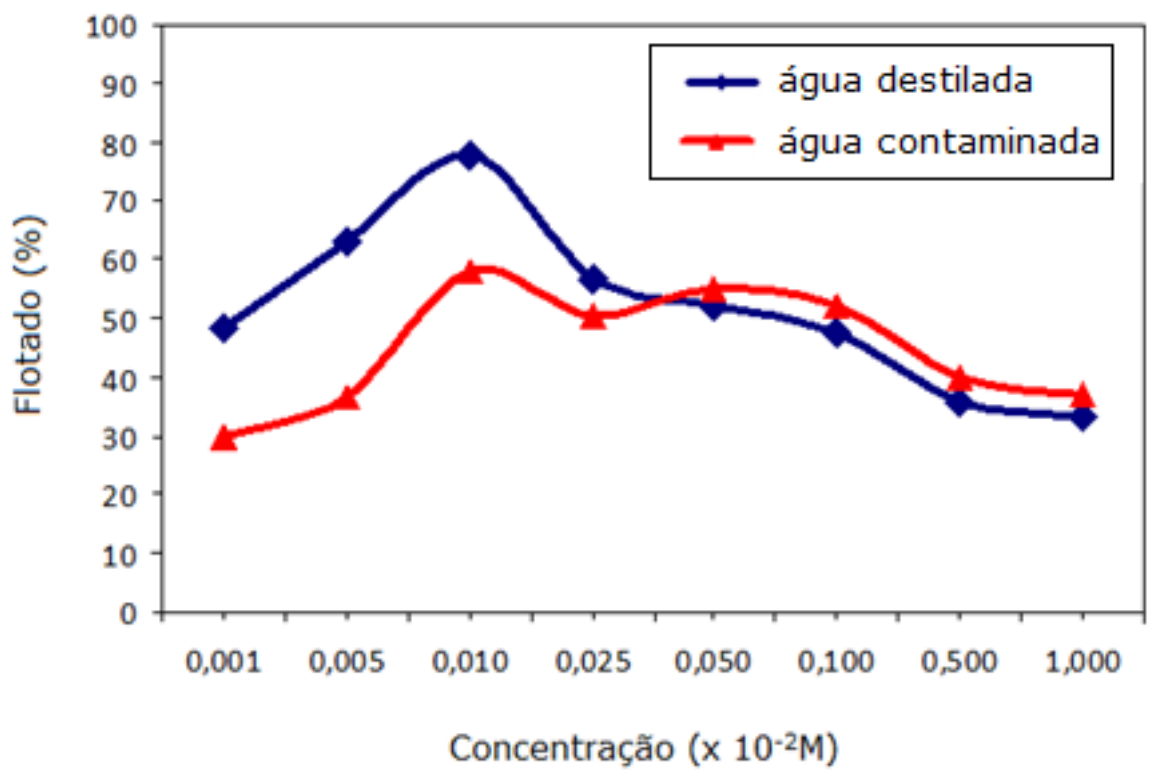

Figura 3 - Influência da concentração de oleato de sódio na recuperação de cromita em função da qualidade da água.

O uso de concentrações elevadas de oleato, no entanto, deve ser evitado devido à intensa espumação provocada por esse tipo de reagente. Dessa forma, verificou-se a possibilidade de diminuir a perda, na recuperação, pela remoção dos eletrólitos por meio de "lavagem" da amostra e renovação da água.

A Figura 4 mostra a redução da condutividade obtida após cada uma das seis "lavagens" realizadas na amostra. Os resultados indicam uma redução de $318 \mu \mathrm{S} / \mathrm{cm}$ para $106,5 \mu \mathrm{S} / \mathrm{cm}$, após a primeira "lavagem". A condutividade seguiu diminuindo até atingir um 
valor de $80,4 \mu \mathrm{S} / \mathrm{cm}$ depois da quinta "lavagem".

A influência da condutividade da polpa na flotação da cromita com oleato é mostrada na Figura 5. Observa-se que a recuperação da cromita aumenta com a remoção dos eletrólitos. Com a água original, enriquecida de eletrólitos pelo contato com o minério, a recuperação foi de apenas $34,3 \%$, subindo sucessivamente após cada etapa de "lavagem" para $48,3 \%, 53,4 \%$ e $56,2 \%$. Com a água destilada obteve-se a recuperação máxima (para as condições testadas) de $77,5 \%$.

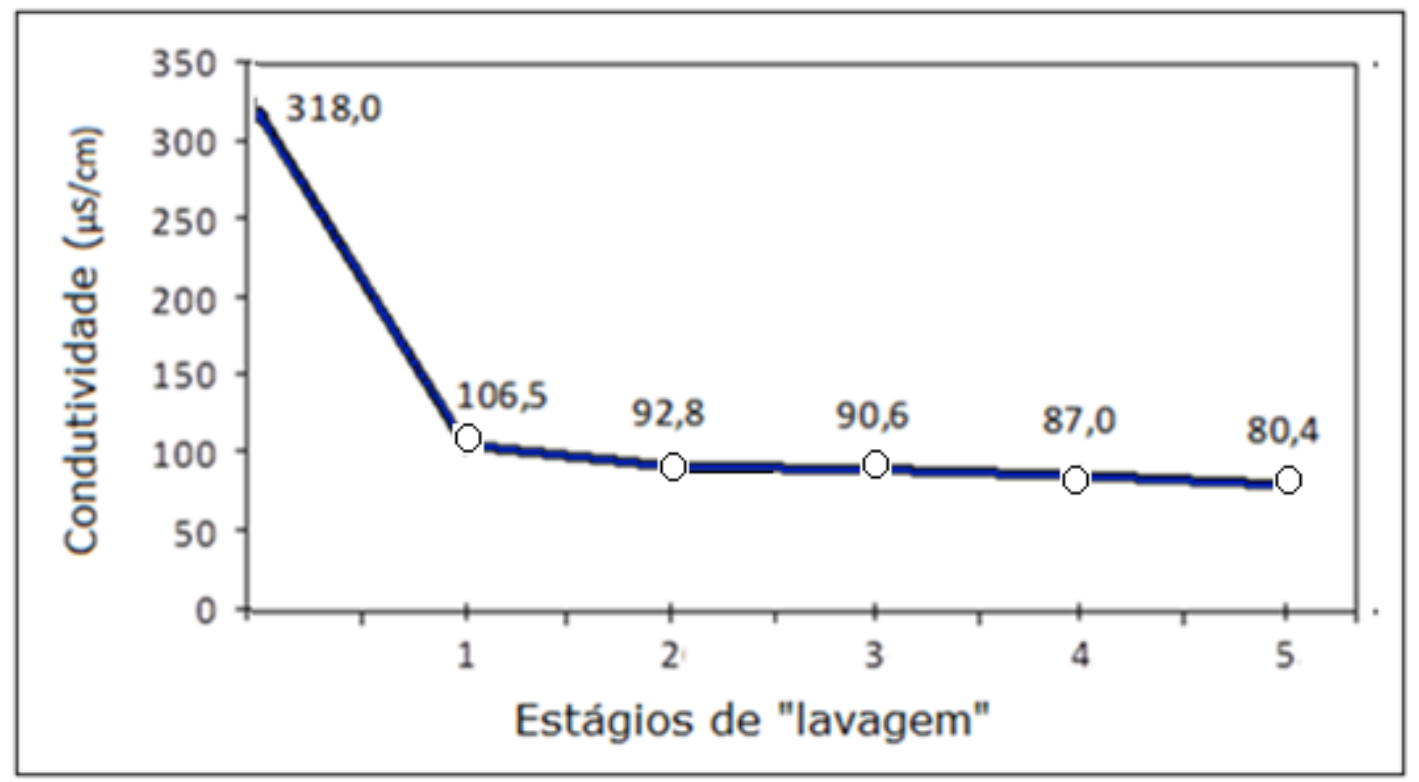

Figura 4 - Redução da condutividade obtida após cada "lavagem" da amostra.

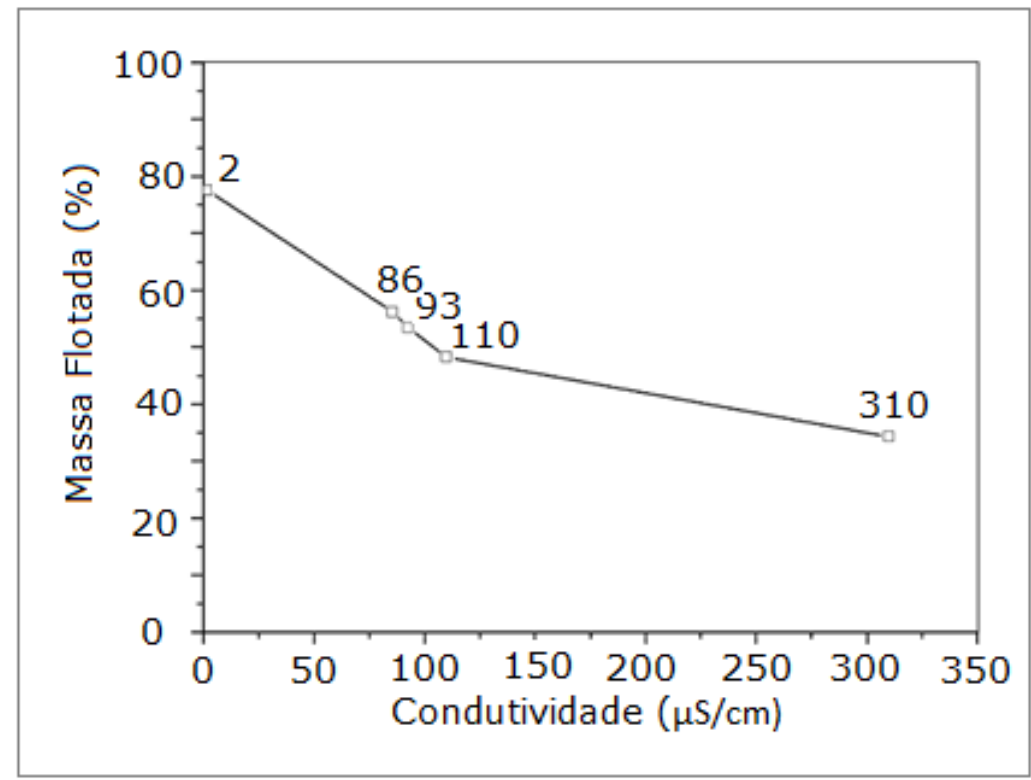

Figura 5 - Influência da quantidade de eletrólitos na polpa na flotação da cromita. 


\section{CONCLUSÕES}

Estudou-se a flotação de cromita com oleato de sódio e a influência das espécies iônicas dissolvidas na seletividade cromita-ganga.

A liberação de íons pela cromita e, especialmente, pelos minerais de ganga é intensa no início do período de contato com a água. A velocidade de dissolução diminui à medida que o contato prossegue.

As espécies catiônicas, provenientes das superfícies minerais, formam sais com o coletor carboxílico. Isso implica na diminuição da disponibilidade de íons oleato na polpa e, consequentemente, na diminuição da densidade de adsorção na superfície.

A ação depressora dos eletrólitos é evidente para pequenas concentrações do coletor (até $10^{-4} \mathrm{M}$ ) provocando uma redução de até $42 \%$ de redução na recuperação da cromita.

O aumento da concentração do oleato anula o efeito depressor dos eletrólitos, mas esse tipo de coletor provoca grande espumação em concentrações elevadas.

A concentração de eletrólitos pode ser reduzida a um nível aceitável por meio de "lavagens" do minério para a remoção das espécies dissolvidas. A recuperação da cromita aumenta à medida que ocorre a remoção dos eletrólitos.

\section{AGRADECIMENTO}

Os autores agradecem ao CNPq pelo apoio financeiro ao projeto "Aproveitamento de Finos de Cromita por Flotação" e pelas bolsas de mestrado a dois dos autores.

\section{REFERÊNCIAS BIBLIOGRÁFICAS}

1. BALTAR, C.A.M.; CUNHA, A.S.F. Influência de espécies catiônicas na flotação de feldspato com amina. In.: Encontro Nacional de Tratamento de Minérios e Metalurgia Extrativa, Anais, v1. BALTAR, C.A.M.; BARBOSA, J.P. e OLIVEIRA, J,C.S. (editores). Recife, p. 234-240, 2002.

2. BALTAR, C.A.M. Flotação no Tratamento de Minérios. 2a edição, Editora Universitária da UFPE, 238 p., 2010.

3. NANTHAKUMAR, B.; GRIMM, D.; PAWLIK, M. Anionic flotation of high-iron phosphate ores - Control of process water chemistry and depression of iron minerals by starch and guar gum. International Journal of Mineral Processing, 92, p. 49-57, 2009.

4. PINHEIRO, V.S.; BALTAR, C.A.M.; LEITE, J.Y.P. Influência da qualidade da água na flotação de quartzo com amina. HOLOS, 3, p. 28-36, 2010.

5. QUINN, J.J.; KRACHT, W.; GOMEZ, C.O.; GAGNON, C.; FINCH, J.A. Comparing the effect of salts and frother (MIBC) on gas dispersion and froth properties. Minerals Engineering, 20, p. 1296-1302, 2007.

5. RAO, S.R.; ESPINOSA-GOMEZ, R.; FINCH, J.A.; BISS, R. Effects of water chemistry on the flotation of pyrochlore and silicate minerals. Minerals Engineering, 1 (3), 189-202. 